\title{
Hidrógeno como combustible complementario para motores de ciclo diésel
}

\section{Hydrogen as a complementary fuel for diesel cycle engines}

\author{
BENITEZ-CRUZ, Gerardo Alan†, CRUZ-GÓMEZ, Marco Antonio*, JUÁREZ-ZERÓN, Tomás Aáron \\ y FLORES-MARTINEZ, Guillermo
}

Benemérita Universidad Autónoma de Puebla

ID $1^{\mathrm{er}}$ Autor: Gerardo Alan, Benitez-Cruz / ORC ID: 0000-0002-0250-3918, Researcher ID Thomson: G-2657-2019, CVU CONACYT ID: 972789

ID $1^{\text {er }}$ Coautor: Marco Antonio, Cruz-Gómez / ORC ID: 0000-0003-1091-8133, Researcher ID Thomson: S-3098-2018, CVU CONACYT ID: 349626

ID $2^{\text {do }}$ Coautor: Tomás Aáron, Juárez-Zerón / ORC ID: 0000-0002-9796-0540, Researcher ID Thomson: S-3099-2018, CVU CONACYT ID: 295058

ID $3^{\text {er }}$ Coautor: Guillermo, Flores-Martinez / ORC ID: 0000-0002-2243-2379, Researcher ID Thomson: G-3384-2019, CVU CONACYT ID: 169853

DOI: $10.35429 / J R E .2019 .11 .3 .15 .22$

Recibido 09 de Octubre, 2019; Aceptado 30 Noviembre, 2019

\begin{abstract}
Resumen
El uso de hidrógeno como combustible complementario en sistemas de combustión interna pretende obtener máquinas automotoras con parámetros propios para el desarrollo sustentable, proporcionando la cantidad de hidrógeno necesaria para ser utilizada en motores de ciclo diésel. El objetivo de esta investigación es cuantificar la cantidad de hidrógeno producida por un prototipo de celda seca para abastecer de forma óptima el suministro energético de un motor diésel en condiciones de operación estándar. Esta investigación fue realizada bajo un enfoque mixto, empleando un procedimiento experimental, sistemático, descriptivo, explicativo, correlacional, empírico y en un contexto de campo, las variables consideradas para el desarrollo de la investigación son las cantidades de hidrógeno, diésel, oxígeno, tiempo y concentración de la mezcla para los experimentos. El prototipo presentó condiciones de operación controladas en función del caudal suministrado de hidrógeno para obtener las curvas de operación del motor a diésel (presión-volumen, temperatura-entropía), estableciendo la concentración necesaria de la mezcla para obtener un rendimiento óptimo para el ciclo de combustión interna. La evolución de los sistemas de combustión con hidrógeno tenderá a optimizar el rendimiento al caracterizar el suministro de manera controlada en función del torque en motores a diésel en condiciones reales de operación.
\end{abstract}

Ciclo diésel, Hidrógeno, Electrólisis

\begin{abstract}
The use of hydrogen as a complementary fuel to internal combustion systems search to obtain automotive machines with individual parameters for sustainable development, providing the hydrogen amount required to be used in diesel cycle engines. The aims of this investigation are to use a dry hydrogen cell prototype to quantify the amount released that is optimal for energetic supply to the diesel cycle in standard operating conditions. This research was conducted under a mixed approach, using an experimental, systematic, descriptive, explanatory, correlational, empirical and in a field context, the variables considered for the development of the research are the amounts of hydrogen, diesel, oxygen, time and concentration of the mixture for the experiments. The prototype presented controlled operating conditions depending on the delivered hydrogen flow to obtain the operating curves of the diesel engine (pressure-volume, temperature-entropy), setting the necessary concentration of the mixture for optimum performance for the internal combustion cycle. The combustion systems evolution with hydrogen will tend to optimize performance when characterizing the supply in a controlled manner, depending on the torque in diesel engines under real operating conditions.
\end{abstract}

Diesel cycle, Hydrogen, Electrolysis

Citación: BENITEZ-CRUZ, Gerardo Alan, CRUZ-GÓMEZ, Marco Antonio, JUÁREZ-ZERÓN, Tomás Aáron y FLORESMARTINEZ, Guillermo. Hidrógeno como combustible complementario para motores de ciclo diésel. Revista de Energías Renovables 2019. 3-11: 15-22

*Correspondencia al Autor (Correo Electrónico: mangcruz@live.com)

$\dagger$ Investigador contribuyendo como primer autor. 


\section{Introducción}

En la actualidad, es posible visualizar los cambios en el ambiente que son resultado del uso de combustibles fósiles y/o de actividades humanas que han llevado a una situación crítica en el medio.

Los gobiernos están cada vez más conscientes del problema que representa seguir dependiendo de combustibles contaminantes, debido a esto se requiere investigar sobre fuentes de energía limpias o en su defecto, que disminuyan las emisiones de los equipos existentes para decrementar el impacto que tienen sobre la naturaleza.

En el caso de continuar con el ritmo actual en que se emiten gases y partículas contaminantes al ambiente, la calidad de vida a mediano y largo plazo se verá sumamente afectada y resultará mucho más complicado tratar de combatir el problema.

Por ello la búsqueda de formas menos contaminantes de obtener energía es imprescindible para continuar con el desarrollo de la sociedad en general y así evitar problemáticas mayores. Se han implementado diversas tecnologías para lograr este objetivo, tales como las celdas fotovoltaicas, vehículos eléctricos o híbridos y motores impulsados con hidrógeno.

El hidrógeno es una de las mejores alternativas para ser el combustible que haga posible la disminución del empleo de combustibles fósiles, entre sus características tenemos:

- Es el elemento más abundante de la naturaleza, pero no existe en estado puro.

- $\quad$ Tiene un alto potencial energético.

- Como combustible, es un gas incoloro, inodoro, insípido y no tóxico.

- $\quad$ Es el más ligero de los gases, su densidad es de $0.0899 \mathrm{~kg} / \mathrm{m}^{3}$ a temperatura $\mathrm{y}$ presión normales.

- $\quad$ Su poder calorífico es de $23 \mathrm{Kcal} / \mathrm{mol}$, mayor al del gas natural $(12 \mathrm{Kcal} / \mathrm{mol}) \mathrm{y}$ la gasolina $(11 \mathrm{Kcal} / \mathrm{mol})$.

- No hay emisiones de $\mathrm{CO}_{2}$ en su combustión, por lo tanto, reduce considerablemente los efectos contaminantes de las máquinas.
Cabe resaltar que el hidrógeno debe obtenerse de otras materias primas (agua, biomasa, combustibles fósiles) y a través de una serie de transformaciones en las que se consume alguna fuente de energía primaria nuclear (electrólisis, termólisis), renovable (gasificación, electrólisis) o fósil (oxidación de gas natural, reformación de gas natural, gasificación).

Esto representa un proceso de obtención que contamina, pero que, a comparación de los demás combustibles existentes, el impacto es mínimo al obtenerlo y al consumirlo.

La investigación para optimizar el uso de hidrógeno como combustible complementario para la operación de distintos tipos de maquinaria que requieren de combustibles fósiles es de vital importancia para ayudar a combatir los efectos negativos del uso de los equipos, así como mejorar la relación costobeneficio y propiciar la tendencia hacia el uso de energías renovables y/o menos contaminantes.

Contar con el uso de hidrógeno como combustible, puede reducir considerablemente la demanda de combustibles fósiles, es por ello que en este artículo se implementó un sistema de cuantificación de producción hidrógeno de bajo costo, en prototipos de celda seca para obtención de hidrógeno por medio de electrólisis, utilizando un sensor MQ-8, Arduino UNO y el software Arduino para programar dicha placa y realizar las mediciones de la producción de hidrógeno, que se obtuvo de una mezcla entre agua destilada e hidróxido de potasio $(\mathrm{KOH})$. Al tener conocimiento de la cantidad de hidrógeno producida por las celdas, podemos establecer el suministro que llega al motor diésel que se desea mantener en operación.

Para poder producir hidrógeno se utilizan prototipos de celda seca, cuya construcción y funcionamiento consta de un cátodo y un ánodo sumergidos en un electrolito conductor y que se les suministra corriente continua proveniente de una batería de $12 \mathrm{~V}$ a 450 cca. Los iones de hidrógeno fluyen hacia el cátodo y los iones de hidróxido a el ánodo, y se utilizan receptores de gas para recoger la producción de hidrógeno y oxígeno formados por la electrólisis. Para la generación de hidrógeno con agua alcalina se usa hidróxido de potasio como electrolito, esto evita la corrosión en los componentes de la celda y porque tiene una conductividad más alta que el hidróxido de sodio $(\mathrm{NaOH})$ que también puede utilizarse para realizar la electrólisis.

BENITEZ-CRUZ, Gerardo Alan, CRUZ-GÓMEZ, Marco Antonio, JUÁREZ-ZERÓN, Tomás Aáron y FLORES-MARTINEZ, Guillermo. Hidrógeno como combustible complementario para motores de ciclo diésel. Revista de Energías Renovables 2019. 
Fuente de alimentación

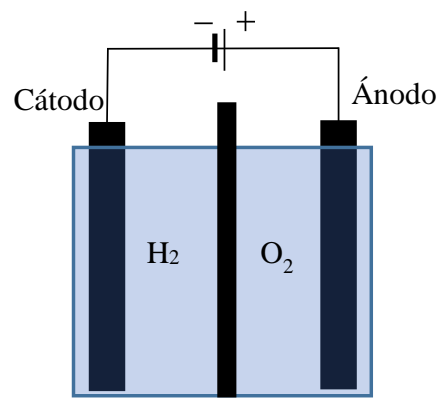

Figura 1 Esquema de unidad de electrólisis del agua Fuente: Elaboración Propia

Cuando se ejecuta la prueba con un electrolito alcalino, las reacciones resultantes en el cátodo y en el ánodo están descritas por las ecuaciones 1 y 2 :

$$
\begin{aligned}
& 2 \mathrm{H}_{2} \mathrm{O}+2 e^{-} \rightarrow \mathrm{H}_{2}+2 \mathrm{OH}^{-}\left(E^{0}=-0.83 \mathrm{~V} \text { vs.SHE } * *\right) \\
& 22 \mathrm{OH}^{-} \rightarrow \frac{1}{2} \mathrm{O}_{2}+\mathrm{H}_{2} \mathrm{O}+2 e^{-}\left(E^{0}=-0.40 \mathrm{~V} \text { vs.SHE } * *\right)
\end{aligned}
$$

Al sumar las ecuaciones 4 y 5 se conduce a la reacción general descrita en la ecuación 3 .

$$
\begin{aligned}
& \mathrm{H}_{2} \mathrm{O} \rightarrow \mathrm{H}_{2}+\frac{1}{2} \mathrm{O}_{2} \\
& \left(E^{0}=-1.23 \mathrm{~V}\right. \text { vs. SHE *) }
\end{aligned}
$$

**Standard hydrogen Electrode (SHE) es la referencia a partir de la cual se determinan todos los potenciales redox estándar.

El comportamiento de las sustancias dentro de los electrodos está indicado por las leyes de Faraday.

En esta investigación, uno de los objetivos es operar un motor diésel MERLIN2 con propósito experimental. El ciclo diésel se lleva a cabo con los siguientes procesos:

1-2. Compresión isentrópica: el aire contenido en el cilindro-émbolo se comprime, disminuyendo el volumen y generando un aumento en la presión y la temperatura.

2-3. Suministro de calor a presión constante por la combustión de la mezcla airecombustible.

3-4. Expansión isoentrópica: el volumen aumenta, disminuyendo la presión y la temperatura, generando trabajo mecánico.

4-1. Rechazo de calor a un volumen constante con disminución de temperatura y presión.

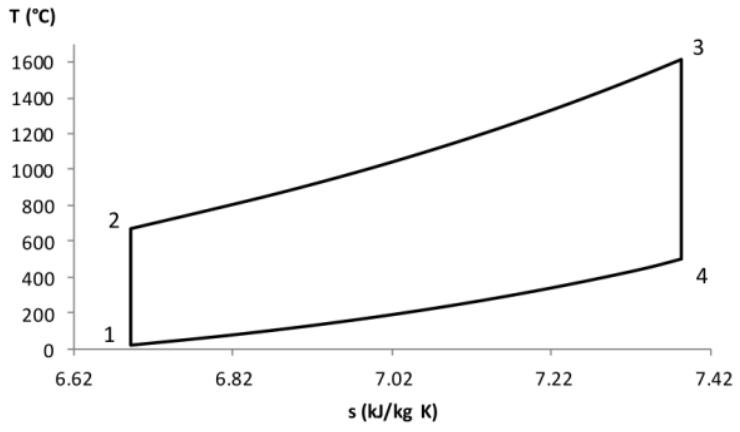

Figura 2 Diagrama temperatura (T)- entropía (s) de ciclo diésel

Fuente: http://148.206.53.84/tesiuami/UAMI19131.pdf

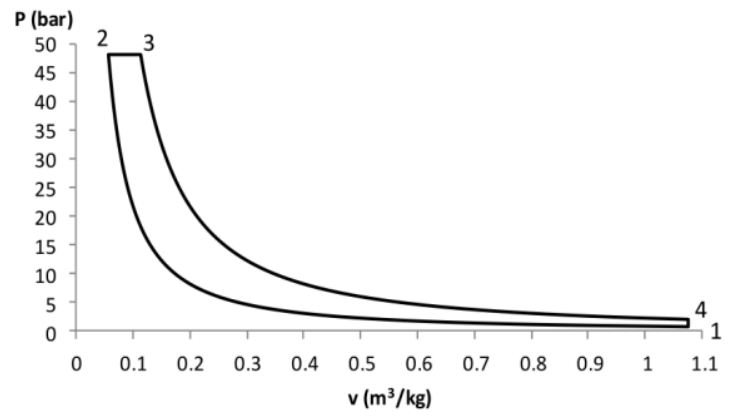

Figura 3 Diagrama presión (P) - volumen (v) de ciclo diésel.

Fuente: http://148.206.53.84/tesiuami/UAMI19131.pdf

El hidrógeno generado se suministra al motor para mantenerlo en operación, teniendo así la posibilidad de funcionar con diésel e hidrógeno, reduciendo el consumo de diésel y por ende economizando costos de operación y emitiendo menor cantidad de contaminantes al ambiente.

\section{Objetivos}

- Desarrollar un sistema de bajo costo capaz de cuantificar la producción de hidrógeno por celdas secas.

- Suministrar hidrógeno y gasóleo a un motor de ciclo diésel para mantenerlo en operación.

- Establecer referencias para futuras investigaciones relacionadas a la producción y uso de hidrógeno como combustible complementario.

- Contribuir al desarrollo de tecnología que se incline hacia el desarrollo sustentable y a la conservación del ambiente. 


\section{Metodología}

Para el desarrollo del proyecto se siguió un enfoque mixto, empleando un procedimiento experimental, sistemático, descriptivo, explicativo, correlacional para manejar las variables, empírico y en un contexto de campo.

La hipótesis formulada para el propósito de la investigación fue de tipo correlacional y causal, adecuada para la relación entre las variables involucradas en el proyecto, y así poder establecer un nivel predictivo y explicativo sobre los efectos al manejar las variables establecidas. La ejecución se llevó a cabo por un diseño explicativo secuencial y los instrumentos utilizados para monitorear y controlar las variables fueron el sistema desarrollado para la cuantificación de la producción de hidrógeno y una báscula de precisión para los experimentos realizados, y así analizar el rendimiento de las conexiones entre las celdas secas y la operación del motor.

Los alcances de esta investigación correspondientes a la parte cuantitativa del enfoque mixto, al combinar la parte experimental, descriptiva, correlacional y explicativa permiten lograr una visualización directa del manejo de las variables y la comprensión del comportamiento de los experimentos, los cuales pueden ser reanalizados posteriormente modificando las variables controladas en la ejecución de la investigación.

Se utilizaron prototipos de celda seca para la producción de hidrógeno, conectadas a una batería de $12 \mathrm{~V}, 450 \mathrm{cca}$, el fluido suministrado a las mismas fue una mezcla de agua destilada e hidróxido de potasio (25gr. De $\mathrm{KOH}$ por cada $400 \mathrm{ml}$ de agua).

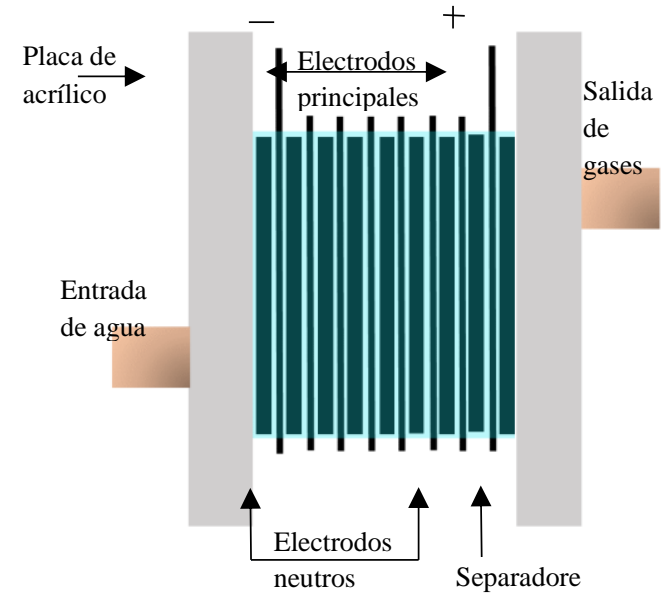

Figura 4 Esquema de celda seca para producción de hidrógeno
Se realizaron experimentos probando distintas conexiones entre las celdas, la producción de hidrógeno varía de acuerdo con el acomodo de estas, ya sea en serie o en paralelo, y se realizaron las mediciones con la ayuda del sensor MQ-8, que registra exclusivamente el hidrógeno que pasa a través de él.
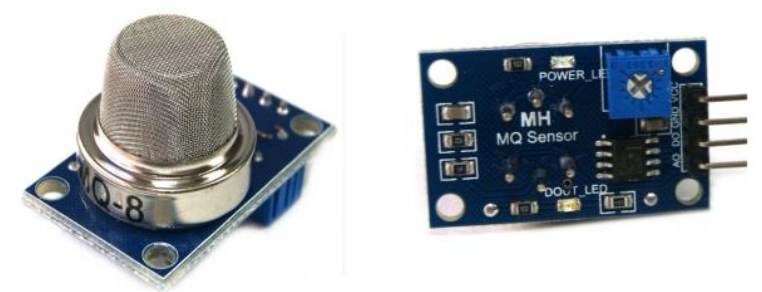

Figura 5 Sensor MQ-8 para detección de hidrógeno Fuente: $\quad$ https://hetpro-store.com/sensor-de-gas-mq-8mq8-h2-hidrogeno/

Para la cuantificación de la producción de hidrógeno es necesario conectar el sensor MQ-8 a una placa Arduino, en este caso se realizó la conexión a una placa Arduino UNO, y a su vez, la placa se conecta a un ordenador para visualizar las lecturas del sensor. La conexión del sensor a la placa se lleva a cabo mediante jumpers macho-macho, y la placa se conecta al ordenador mediante un cable USB. Para visualizar los datos y correr el programa escrito para el muestreo de datos del sensor, dentro del software Arduino se utiliza la herramienta "Monitor serie".

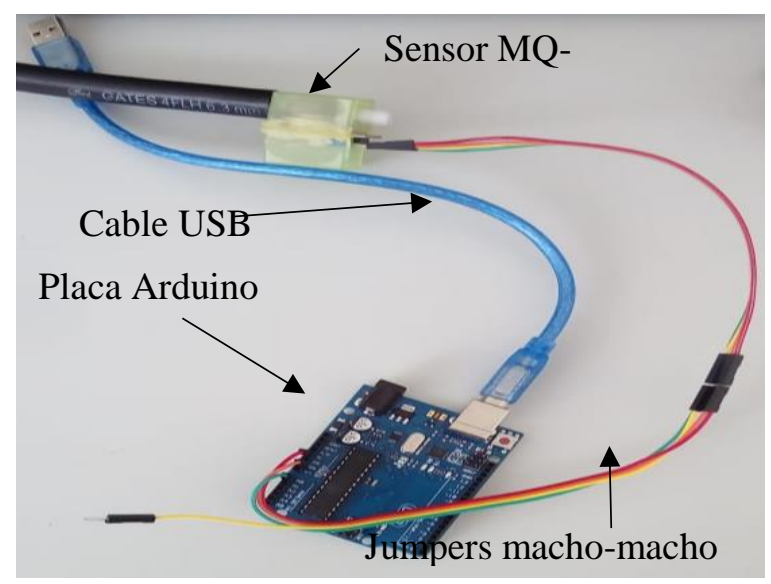

Figura 6 Conexión de sensor MQ-8 a Arduino UNO

\section{Prueba y cuantificación de producción de hidrógeno con celdas en conexión serie}

Los experimentos con las celdas consistieron en probar conexiones entre las mismas para obtener una mayor cantidad de producción de hidrógeno, la primera conexión realizada fue "en serie", obteniendo una cantidad aproximada de $15 \mathrm{ml}$, cuando el sistema de producción se satura (se desaloja el aire atmosférico contenido en el sistema de producción de hidrógeno).

BENITEZ-CRUZ, Gerardo Alan, CRUZ-GÓMEZ, Marco Antonio, JUÁREZ-ZERÓN, Tomás Aáron y FLORES-MARTINEZ, Guillermo. Hidrógeno como combustible complementario para motores de ciclo diésel. Revista de Energías Renovables 2019. 


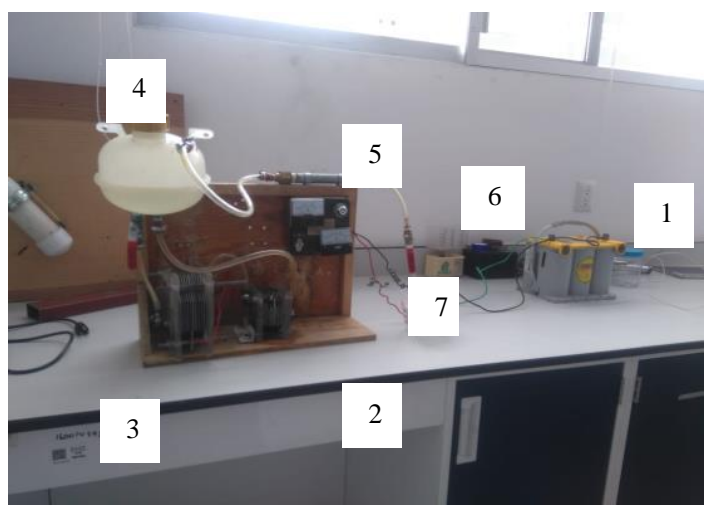

Figura 7 Celdas secas conectadas en serie. (1: batería 12V 450cca, 2 y 3 : Celdas secas, 4: recipiente de balance de 1.6 1t, 5: supresor de flamas, 6: manguera de salida de hidrógeno, 7: voltímetro y amperímetro.)

Fuente: Elaboración Propia

La unión de las celdas nos proporciona un caudal mayor de producción que si se trabaja cada una por separado, en esta primera prueba se conectó el sensor a la salida de hidrógeno del sistema para registrar la cantidad de hidrógeno producida por las celdas. Se energiza el sistema con la batería de $12 \mathrm{~V}$, y por la variación de la carga de esta, las lecturas registradas en el amperímetro y voltímetro fueron de $9 \mathrm{~A}$ y $6 \mathrm{~V}$. La producción oscila entre los $14-15 \mathrm{ml}$ cuando la mezcla entre agua destilada y $\mathrm{KOH}(400 \mathrm{ml}$ de agua por cada $25 \mathrm{~g}$ de $\mathrm{KOH}$ ) se satura y la producción comienza a ser mayor en este punto, y es el tope de producción posible con las celdas secas que se cuentan empleando la conexión en serie, el tiempo para el experimento fue de 7 minutos. Los resultados de las mediciones se muestran en la figura 8.

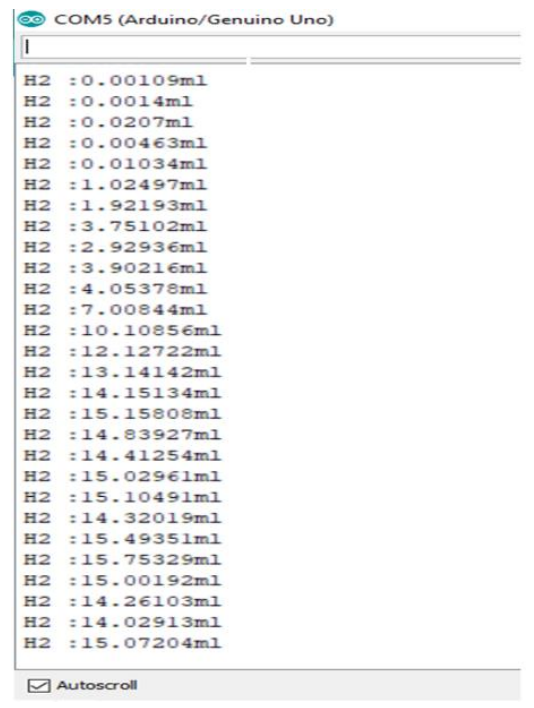

Figura 8 Producción de hidrógeno con celdas secas en serie.

Fuente: Elaboración Propia

El esquema de conexión de las celdas se muestra en la figura 9 .

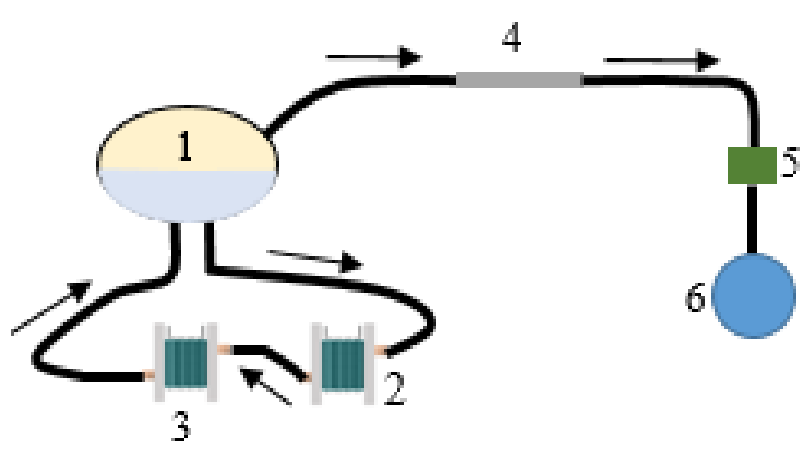

Figura 9 Esquema de conexión de celdas secas en serie (1: recipiente de balance, 2 y 3: celdas secas, 4: supresor de flamas, 5: sensor MQ-8, 6: salida de producción de hidrógeno.)

Fuente: Elaboración Propia.

\section{Prueba y cuantificación de producción de hidrógeno con celdas en conexión en paralelo}

Para la segunda prueba se realizó una conexión en paralelo de las celdas; la producción de hidrógeno por electrolisis del agua fue mayor utilizando esta conexión, se emplean conexiones tipo " $T$ " para unir las mangueras de las celdas $y$ abrazaderas para evitar fugas de fluidos. La conexión en paralelo y su esquema se muestran en las figuras 10 y 11 respectivamente.

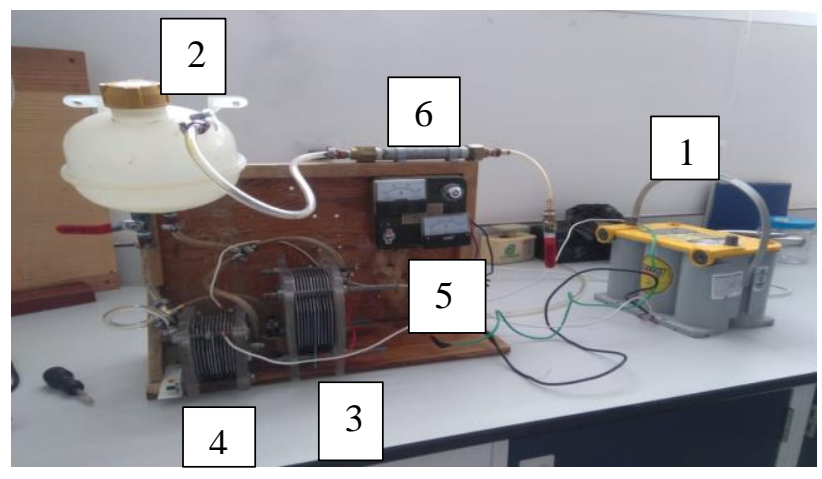

Figura 10 Conexión de celdas secas en paralelo (1: batería $12 \mathrm{~V} 450 \mathrm{cca}, 2$ : recipiente de balance, 3 y 4 : celdas secas, 5: voltímetro y amperímetro, 6: supresor de flamas).

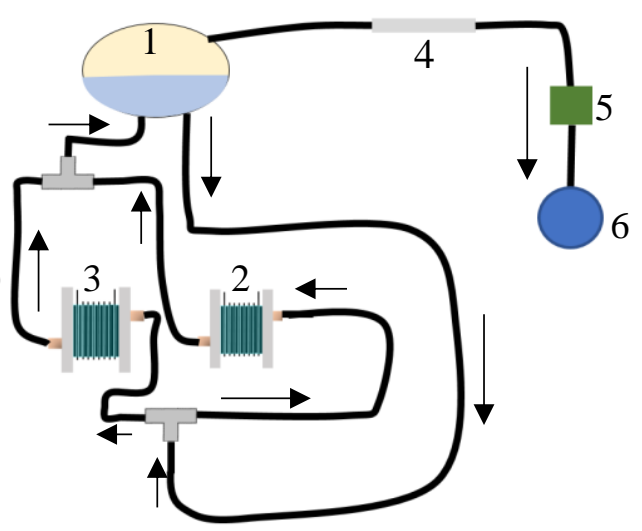

Figura 11 Esquema de conexión de celdas secas en paralelo (1: recipiente de balance, 2 y 3 : celdas secas, 4 : supresor de flamas, 5: sensor MQ-8, 6: salida de producción de hidrógeno.)

BENITEZ-CRUZ, Gerardo Alan, CRUZ-GÓMEZ, Marco Antonio, JUÁREZ-ZERÓN, Tomás Aáron y FLORES-MARTINEZ, Guillermo. Hidrógeno como combustible complementario para motores de ciclo diésel. Revista de Energías Renovables 2019. 
Una vez conectados, se procedió a realizar las mediciones de la generación de hidrógeno, energizando el sistema con la batería, en el amperímetro y voltímetro marcaron lecturas de $6^{\mathrm{a}}$ y $9 \mathrm{~V}$ respectivamente; en este caso, se registró una producción mayor respecto a la prueba anterior. La producción cuando la mezcla se encuentra saturada oscila entre los 20$21 \mathrm{ml}$, el tiempo de la prueba fue de 5 minutos. Los resultados de las mediciones se muestran en la figura 12 .

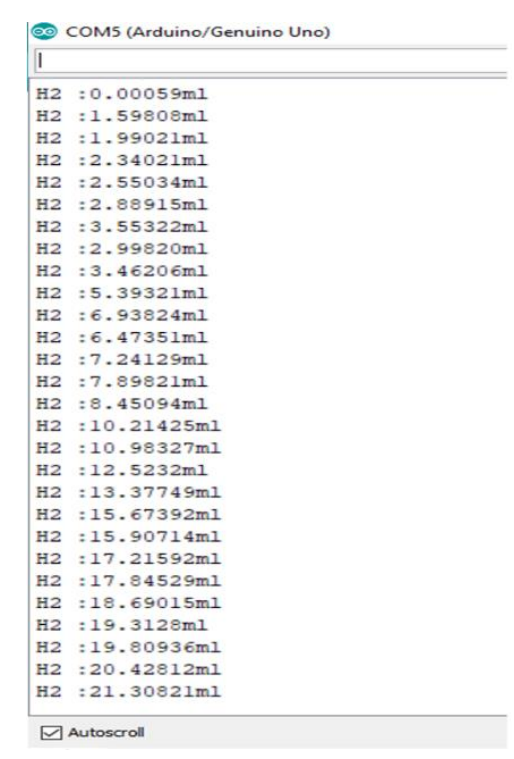

Figura 12 Mediciones de la producción de hidrógeno con la conexión de celdas en paralelo

Fuente: Elaboración Propia

\section{Pruebas en motor de combustión interna con caudal de hidrógeno producido}

Después de cuantificar el hidrógeno obtenido por electrólisis en las celdas secas, se procedió a realizar pruebas con el motor de combustión interna utilizando la conexión en paralelo para tener un suministro mayor del gas hacia el motor.

El experimento consistió en conectar la manguera de salida del hidrógeno a la cuba del motor de ciclo diésel MERLIN2, para tener una fuente de alimentación dual hacia el motor. De esta manera se puede ayudar a reducir el consumo de diésel, decrementado las emisiones contaminantes y el costo de operación del motor. El MERLIN2 es un motor monocilíndrico a 4 tiempos con enfriamiento por aire, opera a una velocidad máxima de 2400 RPM con una potencia generada de alrededor de $1.5 \mathrm{~kW}$, su relación de compresión es de 14.6:1, el volumen de barrido del cilindro es de 0.28 lt. que corresponde a un diámetro de $73 \mathrm{~mm}$ y una carrera de $67 \mathrm{~mm}$.
El motor no arrancó si se alimentaba únicamente con hidrógeno, se tuvo que encender primero consumiendo diésel y pasados unos segundos desde el encendido, se conectaba el suministro de gas hidrógeno para que se mantuviera en operación, fue necesario intercalar la fuente de combustible para que el motor continuara en marcha. Las pruebas realizadas con el motor tuvieron una duración aproximada de 3 minutos.

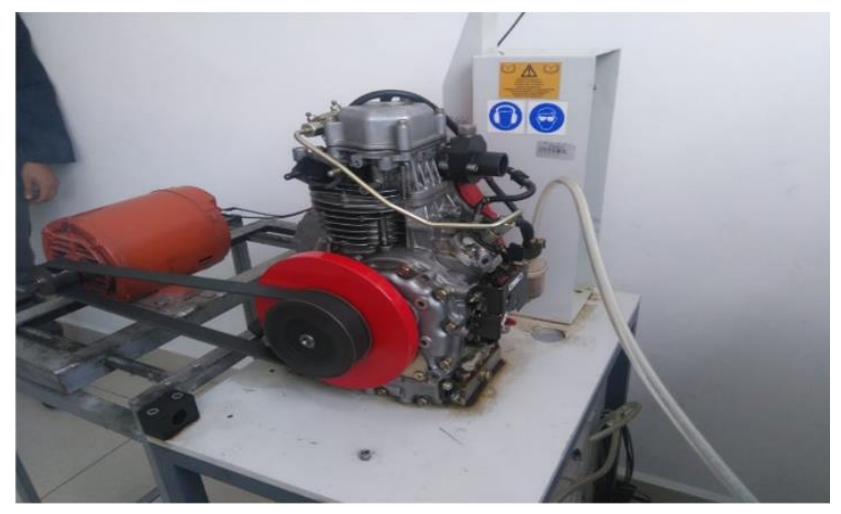

Figura 13 Motor diésel MERLIN2

Fuente: Elaboración Propia

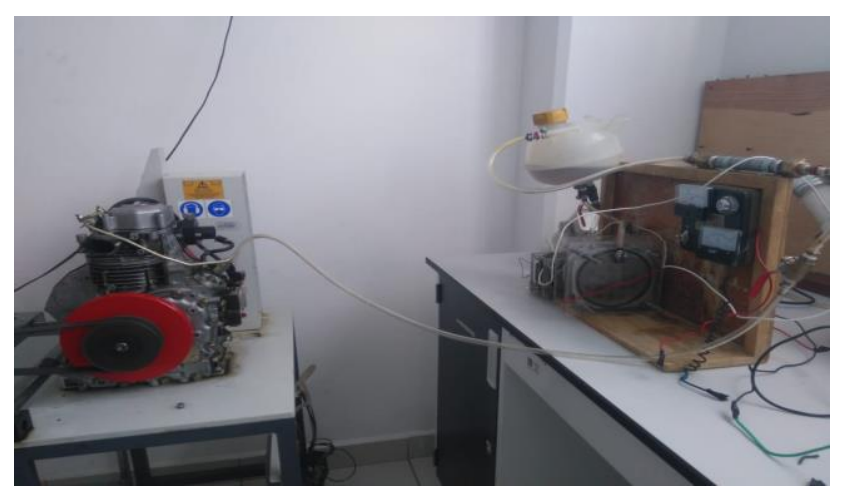

Figura 14 Motor MERLIN2 alimentado con la producción de hidrógeno de celdas secas en conexión paralela Fuente: Elaboración Propia

\section{Resultados}

El sensor MQ-8 y la placa Arduino UNO constituyen un sistema de cuantificación confiable, precisa y de bajo costo para la medición de la producción de hidrógeno que se obtiene de las celdas secas, lo cual permite llevar un registro detallado de los experimentos, obtener datos que permitan mejorar los prototipos y establecer el rango de la eficiencia de la producción de las celdas por medio de métodos estadísticos.

La conexión con mayor eficiencia para la generación del hidrógeno es la unión en paralelo de las celdas, permite el suministro de un caudal mayor en un tiempo de producción menor para poder alimentar a un motor de combustión interna.

BENITEZ-CRUZ, Gerardo Alan, CRUZ-GÓMEZ, Marco Antonio, JUÁREZ-ZERÓN, Tomás Aáron y FLORES-MARTINEZ, Guillermo. Hidrógeno como combustible complementario para motores de ciclo diésel. Revista de Energías Renovables 2019. 
Los efectos negativos del uso de motores de combustión interna, tanto económicos como ambientales, pueden disminuirse con la adecuada implementación de una operación dual en el consumo de combustible entre hidrógeno y combustible fósil, siempre y cuando se suministre la cantidad necesaria de acuerdo a los requerimientos de la operación del motor para evitar paros y/o fallas en el funcionamiento, así como pérdida de la eficiencia del ciclo diésel y de esta manera sea rentable aplicar combustibles poco contaminantes.

\section{Conclusiones}

El método de cuantificación de producción de gas hidrógeno resultante es un sistema preciso y confiable para el registro de los datos, mostrando las lecturas del monitor serie en Arduino (Figuras 8 y 12).

La conexión en paralelo de las celdas secas ofrece una generación de hidrógeno mayor en menor tiempo, sin embargo, se requiere de una fuente de voltaje constante para mantener el proceso de electrólisis en estado óptimo, ya que las baterías se descargan regularmente.

La generación de hidrógeno en las celdas secas puede ayudar a reducir el consumo de combustibles fósiles y disminuir costos de operación y emisiones contaminantes, siendo una de las mejores opciones en la implementación de combustibles renovables.

No obstante, las celdas con las que se contó para el desarrollo de las pruebas requieren de mayor producción de gas hidrógeno para suministrar un caudal adecuado de acuerdo a la demanda del motor, es por ello que debe establecerse un consumo adecuado de combustible dual, entre el diésel y el hidrógeno para operar el motor sin que éste detenga su funcionamiento, puede incrementarse el número de celdas conectadas en paralelo para incrementar la producción de hidrógeno, pero esto requiere mayor espacio para el sistema y también de una fuente de alimentación de mayor tamaño para las celdas secas.

Para que los motores de combustión interna puedan funcionar únicamente con hidrógeno falta mucho por investigar, ya que su producción es costosa y su almacenamiento es difícil de controlar debido a las propiedades del gas.
En los experimentos, el encendido del motor MERLIN2 utilizando únicamente hidrógeno no fue posible, se logró alimentando primero el motor con diésel y una vez encendido se conectó la manguera que proporcionaba el caudal de hidrógeno producido por las celdas para continuar con la operación del motor, fue necesario intercalar el consumo de combustible para mantener en funcionamiento el motor de combustión interna.

\section{Agradecimientos}

- A la Facultad de Ingeniería de la Benemérita Universidad Autónoma de Puebla, por facilitar el acceso a la infraestructura y para el desarrollo del proyecto.

- Al grupo de Tribología y Transporte perteneciente al Cuerpo Académico 189 Prevención de Desastres y Desarrollo Sustentable, Tribología BUAP por su colaboración en el desarrollo de la investigación.

- A la Benemérita Universidad Autónoma de Puebla, por el apoyo al desarrollo del proyecto, así como el acceso a la infraestructura necesaria para completarlo.

- $\quad$ A Antonio Torres José Roberto y Rueda Aguillón Cynthia Janette, por su contribución y apoyo en la ejecución de los experimentos realizados.

\section{Referencias}

Aydin, K., Kenanoglu, R. (2018, abril 26). Effects of hydrogenation of fossil fuels with hydrogen and hydroxy gas on performace and emissions of internal combustión engines. International Journal of Hydrogen Energy, 43, 14047-14058, 2019, junio 11. De ScienceDirect Base de datos.

Castillo, V. (2016) Producción, caracterización y desempeño del biodiesel en un motor diésel con mezclas B5, B10 Y B20 (tesis de maestría) Universidad Autónoma Metropolitana, D.F., México.

Fernández S.. (2009). ¿Por qué hidrógeno?. 2019, abril 22, de DocPlayer Sitio web: http://docplayer.es/docview/54/33905019/\#pag $\mathrm{e}=9$ 
Juárez A. et al. (2018, septiembre). Caracterización de celda para producción de hidrógeno con fin de generar combustible alternativo para motores de combustión interna. Revista de energías renovables, Vol. 2 no. 6, 2632. 2019, abril 30.

Pavlos D., Madan K., Taku T., \& Yasumasa S.. (2018, junio 21). Combustion and emission characteristics of a hydrogen-diesel dual-fuel engine. International Journal of Hydrogen Energy, 43, 13605-13617. 2019, abril 30, De ScienceDirect Base de datos.

Rolle, K.. (2006). Termodinámica. México: Pearson Educación.

Serrano, J., Jiménez-Espadafor, F.J., \& López, A.. (2019, febrero 4). Analysis of the effect of different hydrogen/diesel ratios on the performance and emissions of a modified compression ignition engine under dual-fuel mode with water injection. Hydrogen-diesel dual-fuel mode.. Energy, 172, 702-711. 2019, abril 20, De ScienceDirect Base de datos.

Verma S. et al, (2019, mayo 25). A renewable pathway towards increased utilization of hydrogen in diesel engines, International Journal of Hydrogen Energy, 2019, junio 20. De ScienceDirect Base de datos.

Yilmaz, .I.T, Gumus, M., (2017, octubre 5). Effects of hydrogen adittion to the intake air on performance and emissions of common rail diesel engine. Energy, 142, 1104-1113. 2019, mayo 4. De ScienceDirect Base de datos. 